\title{
Influence of gas rarefaction on the lateral resolution achievable by thermocapillary patterning
}

\author{
Nan Liu and Sandra M. Troian ${ }^{\text {a) }}$ \\ California Institute of Technology, 1200 E. California Blvd., T. J. Watson Sr. Laboratories \\ of Applied Physics, MC 128-95, Pasadena, California 91125, USA
}

(Received 24 September 2010; accepted 13 January 2011; published online 8 February 2011)

\begin{abstract}
Molten polymer nanofilms subject to a large transverse thermal gradient can undergo a thermocapillary instability leading to the growth of nanopillar arrays on the cooler side. The array pitch can be estimated from the fastest growing wavelength from linear stability analysis. Here we quantify the influence of gas rarefaction on thermal conduction for cases in which the gas layer thickness above the film approaches dimensions of the molecular mean free path. For experimentally relevant parameters, rarefaction increases the pitch by as much as $65 \%$, an important consideration for noncontact three-dimensional structure formation by thermocapillary lithography. () 2011 American Institute of Physics. [doi:10.1063/1.3551535]
\end{abstract}

Optical lithography has served as the backbone of the semiconductor industry for decades. Reduction in the source radiation wavelength, increase in lens numerical aperture, enhanced proximity correction algorithms, and improved phase shifting masks have led to the fabrication of structures as small as tens of nanometers. While a few methods can compete with this technological achievement, there is nonetheless emerging interest in developing alternative lithographic techniques compatible not only with silicon or other semiconducting materials but also polymers, colloids, and microstructured materials. ${ }^{1}$ This pursuit is being driven by lower estimates of manufacturing costs from a fewer processing steps as well as the possibility of noncontact patterning methods based on fluid instabilities for producing threedimensional (3D) structures in a single step. It is well known that interfacial instabilities in thin films can generate periodic deformations whose amplitude and growth are typically suppressed by gravitational leveling. ${ }^{2}$ At nanoscale dimensions, however, gravitational forces are negligible in comparison to driving forces for shaping liquid structures by modulation of interfacial stresses. ${ }^{3}$ In this limit, elongated structures become possible, paving the way for fabrication techniques based on interface sculpting. As an added bonus, fluid based techniques tend to produce ultrasmooth surfaces ideally suited to photonic applications since the final structures are obtained directly from in situ solidification of molten shapes.

During the past several years, 3D structure formation in nanoscale polymer melts subject to a large thermal gradient has been reported by several groups. Three growth mechanisms have been proposed including radiation pressure from interface reflection of low frequency acoustic phonons, ${ }^{4,5} \mathrm{im}$ age charge induced electrostatic attraction between the film and target substrate, ${ }^{6}$ and thermocapillary flow directed toward the cooler target. ${ }^{7}$ Recent hydrodynamic studies ${ }^{7,8}$ suggest that a thermocapillary instability is the predominant driving force and that the phenomenon is basic to any viscous nanofilm not just polymer based films. ${ }^{7,8}$ Fluid elongations are caused by a long wavelength Bénard-Marangoni instability in the limit of zero gravity. The wavelength of the fastest growing mode,

${ }^{a)}$ Electronic mail: stroian@caltech.edu.

$$
\lambda_{\max }=2 \pi h_{o} \sqrt{\frac{4 \gamma h_{o}}{3 \kappa \gamma_{T} d_{o} \Delta T}}\left[\frac{d_{o}}{h_{o}}+\kappa-1\right],
$$

establishes the array pitch or resolution limit based on the initial film thickness $h_{o}$ and normalized gap width $d_{o} / h_{o}$; the melt surface tension $\gamma$; the maximum change in surface tension with temperature, $\gamma_{T} \Delta T$, where $\gamma_{T}=|d \gamma / d T|$ and $\Delta T$ $=T_{2}-T_{1}>0$; and the ratio of thermal conductivities in the gas and melt layers, $\kappa=k_{\text {gas }} / k_{\text {melt }}$. With all else equal, a larger thermal gradient yields a smaller array pitch and therefore higher resolution arrays.

Chou et al. ${ }^{10,11}$ and Schäffer et al. ${ }^{4,5}$ first uncovered this instability in polymer nanofilms of polystyrene (PS) and poly(methyl methacrylate) using the experimental setup shown in Fig. 1. The measured amplitudes and pitches ranged from $125 \leqq d_{o} \lesssim 375 \mathrm{~nm}$ and $1 \lesssim \lambda_{\max } \lesssim 10 \mu \mathrm{m}$. In the experiments of Schäffer et al., the initial polymer film was spun cast to a thickness $80 \leqq h_{o} \lesssim 130 \mathrm{~nm}$ onto a silicon wafer. A second wafer with protruding spacers was placed above this film, leaving an air gap of thickness $5 \leq d_{o}-h_{o}$

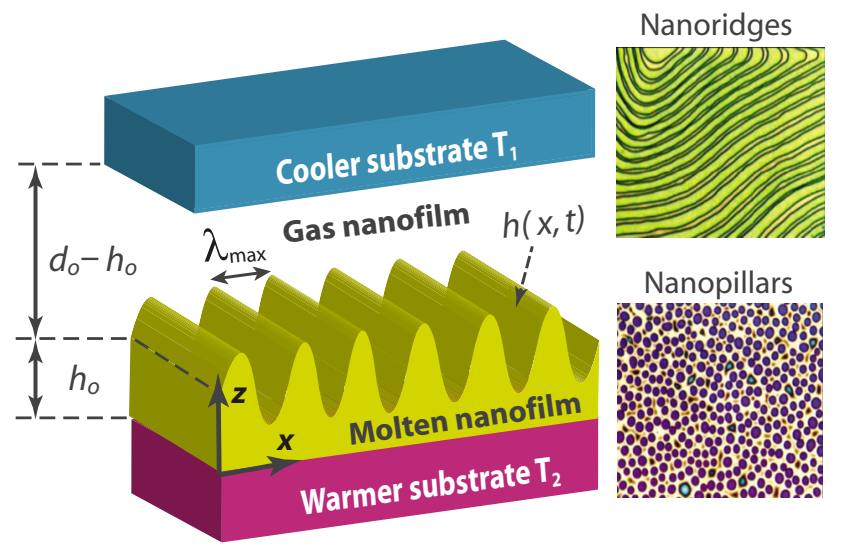

FIG. 1. (Color online) Sketch of experimental setup for generation of lamellar (nanoridges) and nanopillar arrays obtained by subjecting a molten film with initial thickness $h_{o}$ and gas layer thickness $d_{o}-h_{o}$ to a large thermal gradient of order $\left(T_{2}-T_{1}\right) / d_{o} \approx 10^{6}-10^{8}{ }^{\circ} \mathrm{C} / \mathrm{cm}$ (not drawn to scale). Optical micrographs from Ref. 9 showing solidified formations in polystyrene films with $\lambda_{\max } \approx 3-5 \mu \mathrm{m}$ and $d_{o} \approx 108-755 \mathrm{~nm}$. In the theoretical analysis, the variable $h(x, t)$ represents the local thickness of the moving interface at position $x$ at time $t$. 
$\$ 270 \mathrm{~nm}$. The smallest air gaps approached dimensions of the molecular mean free path length $\lambda_{\text {gas }}$. This confinement led to an overall temperature difference $10 \leqq \Delta T \leqq 50^{\circ} \mathrm{C}$ and therefore thermal gradients of order $10^{6}-10^{8}{ }^{\circ} \mathrm{C} / \mathrm{cm}$. The elongated structures grew until contact with the cooler wafer. In situ solidification was achieved by dropping both temperatures below the polymer glass transition. Removal of the top wafer revealed arrays of nanopeaks.

Temperature variations along a fluid interface produce variations in the surface tension which generate a thermocapillary stress $\tau=(d \gamma / d T) \nabla T$. Liquids with a negative value $d \gamma / d T$ therefore flow toward cooler regions. For Knudsen number $\mathrm{Kn}=\lambda_{\text {gas }} /\left(d_{o}-h_{o}\right) \ll 1$, continuum theory holds and the temperature field in between the wafers can be computed from the steady state one-dimensional thermal conduction equation, $\partial^{2} T / \partial z^{2}=0$, subject to isothermal boundary conditions at $z=0$ and $z=d_{o}{ }^{7,8}$ This simplicity arises from the long wavelength (i.e., slender gap) approximation $\left(d_{o} / \lambda_{\max }\right)^{2} \ll 1$, which reduces the three-dimensional analysis to a onedimensional conduction problem along the $z$-axis. Also, since the thermal diffusive time scale is orders of magnitude smaller than the viscous flow time scale, the temperature field within the gap and along the moving interface equilibrates instantaneously. It is well recognized that gas rarefaction effects must be included in the thermal analysis for $\mathrm{Kn} \geq 0.1{ }^{12}$ Rarefaction is especially important, for example, in hard disk drives where accurate determination of the thermal flux is critical to modern thermal flying-height control sliders. ${ }^{13}$ Our estimates based on previous experiments ${ }^{5}$ indicate that $0.3 \leq \mathrm{Kn} \leq 9$. In this letter, we quantify the influence of gas rarefaction on the lateral resolution achievable by thermocapillary patterning for cases in which the gas layer thickness approaches the molecular mean free path.

There exists no analytical form for the heat flux in a rarefied gas due to the formidable difficulty in solving the integro-differential Boltzmann equation. To a good approximation, however, one can use temperature jump conditions $^{14,15}$ at $z=h(x, t)$ and $z=d_{o}$ to determine the thermal flux boundary condition at $z=h(x, t)$, from which the interfacial shear stress can be computed. The jump conditions are given by $T_{h}^{\text {gas }}=T(h)-\Lambda|\partial T / \partial z|_{z=h}$ and $T_{d_{o}}^{\text {gas }}=T\left(d_{o}\right)$ $+\Lambda|\partial T / \partial z|_{z=d_{o}}$. Here, $\Lambda=[(2-\sigma) / \sigma][2 C /(C+1)] \lambda_{\text {gas }} / \operatorname{Pr}$. Material constants refer to the gas phase where $0.85 \leq \sigma$ $\leq 1.0$ is the thermal accommodation coefficient, ${ }^{16}$ which for convenience is assumed to be equal at $z=h$ and $z=d_{o}, C$ $=c_{p} / c_{v}$ is the isoentropic exponent, and $\operatorname{Pr}$ is the Prandtl number. The mean free path length for air molecules according to the variable soft sphere model ${ }^{15}$ is $\lambda_{\text {gas }}$ $=k_{B} T\left(\sqrt{2} \pi d_{\text {ref }}^{2} P\right)^{-1}\left(T / T_{\text {ref }}\right)^{\omega-1 / 2}$, where $k_{B}$ is the Boltzmann constant, $P$ is the pressure, $d_{\text {ref }}$ is the so-called effective diameter at the reference temperature $T_{\text {ref }}$, and $\omega$ is an exponent ranging from $1 / 2$ (hard sphere model) to 1.0 (Maxwell model). For an air film at $T_{\text {ref }}=273 \mathrm{~K}$ and $\omega=0.77, d_{\text {ref }}$ $=0.42 \mathrm{~nm}$. The thermal flux extracted from the interface $z$ $=h(x, t)$ is then

$$
q=k_{\mathrm{air}} \frac{T_{z=h(x, t)}-T_{1}}{d_{o}-h(x, t)+2 \Lambda},
$$

which reduces to the continuum result in the limit $\Lambda \ll d_{o}$ $-h$. Recent studies ${ }^{17}$ using direct simulation Monte Carlo confirm that this approximate form for the thermal flux re-

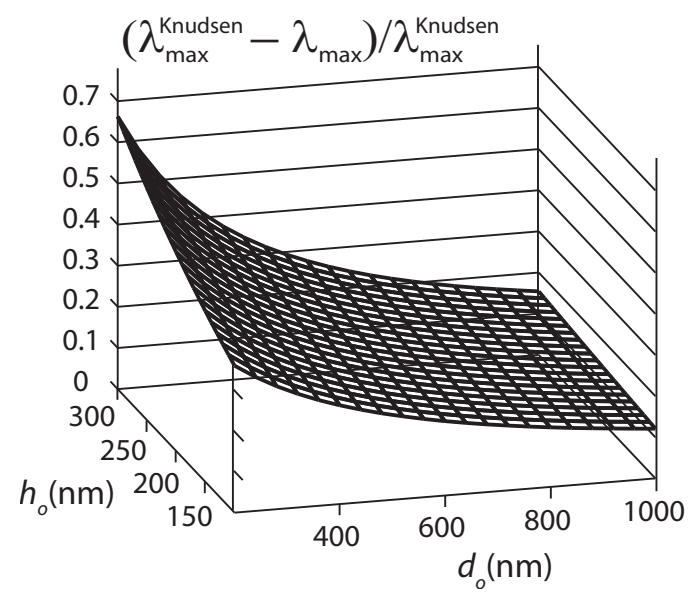

FIG. 2. Relative difference in the fastest growing wavelength with $\left(\lambda_{\max }^{\text {Knudsen }}\right)$ and without $\left(\lambda_{\max }\right)$ gas rarefaction. Parameter values correspond to a PS nanofilm with $T_{2}=170{ }^{\circ} \mathrm{C}, T_{1}=124^{\circ} \mathrm{C}, \mu_{\mathrm{PS}}=2.5 \times 10^{4} \mathrm{~Pa} \mathrm{~s}, \mu_{\text {air }}=2.48$ $\times 10^{-5} \mathrm{~Pa} \mathrm{~s}, \quad k_{\mathrm{PS}}=0.130 \mathrm{~W} / \mathrm{m} \mathrm{K}, \quad k_{\text {air }}=0.036 \mathrm{~W} / \mathrm{m} \mathrm{K}, \quad \gamma=31.5$ $\times 10^{-3} \mathrm{~N} / \mathrm{m}$, and $\gamma_{T}=0.089 \times 10^{-3} \mathrm{~N} / \mathrm{m} \mathrm{K}$, as examined in Refs. 5 and 8 .

mains valid up to $K n \approx 20$ even in the presence of shear flow. Thermal conduction within the molten film subject to Eq. (2) and $T(z=0)=T_{2}$ yields the expression for the interface temperature:

$$
T_{z=h}=\frac{\left(d_{o}-h+2 \Lambda\right) T_{2}+\kappa h T_{1}}{d_{o}+(\kappa-1) h+2 \Lambda} .
$$

The corresponding evolution equation is given by

$$
\frac{\partial h}{\partial t}+\frac{1}{2 \mu} \frac{d \gamma}{d T} \frac{\partial}{\partial x}\left(h^{2} \frac{\partial}{\partial x} T(z=h)\right)+\frac{\gamma}{3 \mu} \frac{\partial}{\partial x}\left(h^{3} \frac{\partial^{3} h}{\partial x^{3}}\right)=0,
$$

where to first $\operatorname{order}^{8}$ the material properties $\mu, \gamma$, and $d \gamma / d T$ are held constant and evaluated at $T_{2}$. The second term in Eq. (4) represents destabilizing shear flow by thermocapillary stresses, which enhances the growth of elongated structures; the third term represents stabilizing capillary flow which represses surface undulations and slows growth. A linear stability analysis can then be applied by subjecting the flat film to an infinitesimal sinusoidal disturbance where $h(x, t)=h_{o}$ $+\delta h(x, t)=h_{o}+a \exp (\beta t+i k x)$, where $a \ll h_{o}$. The function $\beta(k)$ represents the growth rate of the mode with wave number $k=2 \pi / \lambda$ :

$$
\beta(k)=\left\{\frac{\kappa \gamma_{T} \Delta T}{2 \mu} \frac{h_{o}^{2}(d+2 \Lambda)}{\left[d_{o}+(\kappa-1) h_{o}+2 \Lambda\right]^{2}}-\frac{\gamma h_{o}^{3}}{3 \mu} k^{2}\right\} k^{2} .
$$

The wavelength of the fastest growing mode is given by

$$
\frac{\lambda_{\max }^{\text {Knudsen }}}{2 \pi h_{o}}=\sqrt{\frac{4 \gamma h_{o}}{3 \kappa \gamma_{T} \Delta T\left(d_{o}+2 \Lambda\right)}}\left[\frac{d_{o}+2 \Lambda}{h_{o}}+(\kappa-1)\right] .
$$

Comparison of Eqs. (1) and (6) reveals that gas rarefaction effectively augments the gap spacing $d_{o}$ by an amount $2 \Lambda$. Thermal jump conditions in the Knudsen regime produce cooler gas temperatures near the melt interface and warmer gas temperatures near the top wafer. This effect in turn diminishes the actual thermal gradient across the gas layer, thereby diminishing thermocapillary effects. The relative difference in pitch given by Eqs. (1) and (6) is shown in Fig. 2. 


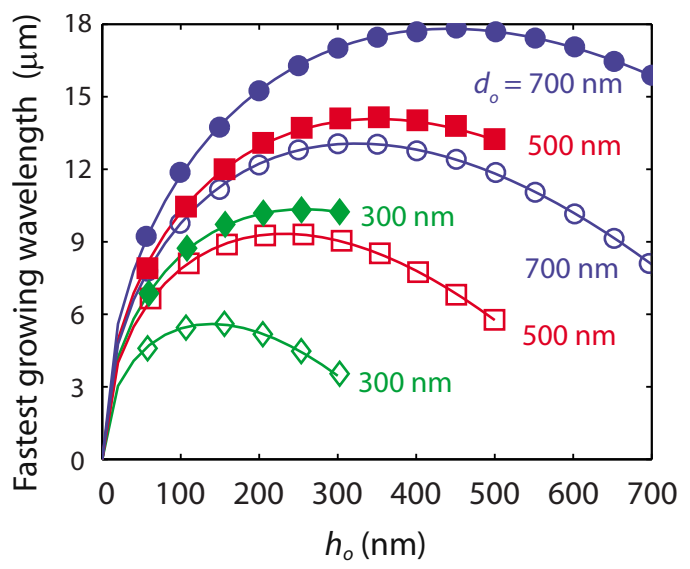

FIG. 3. (Color online) Fastest growing wavelength $\lambda_{\max }$ with (solid symbols) and without (open symbols) rarefaction effects as a function of initial film thickness $h_{o}$ and gap width $d_{o}$. Additional parameter values are given in Fig. 2.

As evident, this ratio increases for larger initial film thicknesses $h_{o}$ at fixed values of $d_{o}$ or smaller gap spacings $d_{o}$ at fixed values of $h_{o}$. For parameter values corresponding to the experiments of Schäffer et al., the relative difference can exceed $65 \%$.

Shown in Fig. 3 is the variation in the fastest growing wavelength with (solid symbols) and without (open symbols) gas rarefaction as a function of the initial film thickness and gap width for the same parameter values used in Fig. 2. From Eq. (5), the net fluid flux resulting from the competition between thermocapillary and capillary stresses generates the local maximum shown. Rarefaction effects yield a larger pitch and therefore lower resolution arrays. However, according to Eq. (6), the pitch can be further reduced by using gases with higher thermal conductivities than air, molten nanofilms with smaller thermal conductivities than polystyrene, larger values of $\Delta T$, reduction in the ratio $d_{o} / h_{o}$, or smaller film thicknesses $h_{o}$. The characteristic growth time for 3D elongations, as estimated from $\beta^{-1}$ in Eq. (5) for $k_{\max }=2 \pi / \lambda_{\max }$, with (solid symbols) and without (open symbols) rarefaction effects is shown in Fig. 4. Rarefaction increases the time needed for structure formation because of the reduction in the effective thermal gradient across the gas layer. This effect is most notable for the smallest ratios $d_{o} / h_{o}$.

In summary, we have shown that gas rarefaction leads to an increase in the array pitch and growth rate for 3D nanostructures formed by thermocapillary patterning. Temperature jump conditions at the boundaries of the confined gas

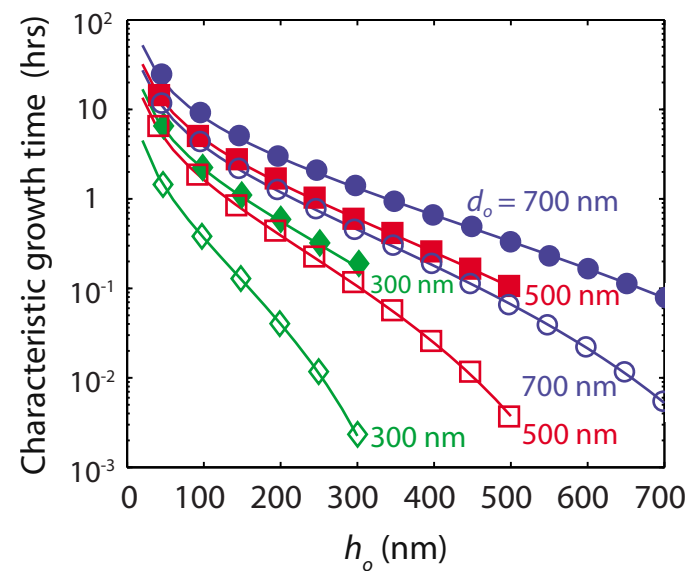

FIG. 4. (Color online) Characteristic growth time for pillar formation, $\beta^{-1}\left(k=k_{\max }=2 \pi / \lambda_{\max }\right)$, with (solid symbols) and without (open symbols) rarefaction effects as a function of initial film thickness $h_{o}$ and gap spacing $d_{o}$. Additional parameter values are given in Fig. 2.

layer lead to a reduction in the effective thermal gradient. The ultimate pitch achievable by this technique can nonetheless be substantially reduced by optimizing the geometric dimensions and material properties according to the expressions provided.

SMT gratefully acknowledges financial support from the National Science Foundation through Grant Nos. CTS 0649474 and CBET 0701324.

${ }^{1}$ A. del Campo and E. Artzt, Chem. Rev. (Washington, D.C.) 108, 911 (2008).

${ }^{2}$ A. Oron, S. H. Davis, and S. G. Bankoff, Rev. Mod. Phys. 69, 931 (1997).

${ }^{3}$ A. A. Darhuber and S. M. Troian, Annu. Rev. Fluid Mech. 37, 425 (2005).

${ }^{4}$ E. Schäffer, S. Harkema, R. Blossey, and U. Steiner, Europhys. Lett. 60, 255 (2002).

${ }^{5}$ E. Schäffer, S. Harkema, M. Roerdink, R. Blossey, and U. Steiner, Macromolecules 36, 1645 (2003).

${ }^{6}$ L. Wu and S. Chou, J. Non-Newtonian Fluid Mech. 125, 91 (2005).

${ }^{7}$ M. Dietzel and S. M. Troian, Phys. Rev. Lett. 103, 074501 (2009).

${ }^{8}$ M. Dietzel and S. M. Troian, J. Appl. Phys. 108, 074308 (2010).

${ }^{9}$ E. Schäffer, Ph.D. thesis, Universität Konstanz, 2001.

${ }^{10}$ S. Y. Chou and L. Zhuang, J. Vac. Sci. Technol. B 17, 3197 (1999).

${ }^{11}$ S. Y. Chou, L. Zhuang, and L. Guo, Appl. Phys. Lett. 75, 1004 (1999).

${ }^{12}$ S. A. Schaaf and P. L. Chambrè, Flow of Rarefied Gases (Princeton University Press, Princeton, NJ, 1961).

${ }^{13}$ N. Liu, J. Zheng, and D. B. Bogy, J. Appl. Phys. 108, 016102 (2010).

${ }^{14}$ E. H. Kennard, Kinetic Theory of Gases (McGraw Hill, New York, 1931).

${ }^{15}$ G. A. Bird, Molecular Gas Dynamics and Direct Simulation of Gas Flows (Clarendon, Oxford, 1994).

${ }^{16}$ M. Knudsen, Ann. Phys. (Berlin) 339, 593 (1911).

${ }^{17}$ Y. S. Ju, J. Heat Transfer 122, 817 (2000) 